\title{
Shock Hugoniot Data for Water up to 5 Mbar Obtained with Quartz Standard at High-Energy Laser Facilities
}

\author{
D. Mancelli $\mathbb{D}^{1,2}$ I. Errea, ${ }^{2,3,4}$ A. Tentori, ${ }^{1}$ O. Turianska, ${ }^{1}$ H. Larreur, ${ }^{1}$ K. Katagiri, ${ }^{5,6}$ \\ N. Ozaki $\mathbb{D}^{5,6}$ N. Kamimura, ${ }^{5}$ D. Kamibayashi, ${ }^{5}$ K. Ishida, ${ }^{5}$ H. Ogura, ${ }^{5}$ K. Kawasaki, ${ }^{6}$ \\ Y. Maeda, ${ }^{6}$ Y. Hironaka, ${ }^{6}$ K. Shigemori, ${ }^{6}$ K. Batani, ${ }^{7}$ G. Schaumann, ${ }^{8}$ O. Rosmej, ${ }^{8}$ \\ P. Neumayer, ${ }^{8}$ B. Zielbauer, ${ }^{8}$ A. S. Martynenko, ${ }^{8,9}$ E. D. Filippov $\mathbb{D}^{9,10}$ S. Pikuz, ${ }^{9,11}$ \\ and D. Batani ${ }^{1,11}$ \\ ${ }^{1}$ Universitè de Bordeaux, CNRS, CEA, CELIA, UMR 5107, F-33405 Talence, France \\ ${ }^{2}$ Donostia International Physics Center (DIPC), Donostia/San Sebastián, Spain \\ ${ }^{3}$ Centro de Física de Materiales (CSIC-UPV/EHU), Donostia/San Sebastián, Spain \\ ${ }^{4}$ Fisika Aplikatua Saila, Gipuzkoako Ingeniaritza Eskola, University of the Basque Country (UPV/EHU), \\ Donostia/San Sebastián, Spain \\ ${ }^{5}$ Graduate School of Engineering, Osaka University, Suita, Osaka 565-0871, Japan \\ ${ }^{6}$ Institute of Laser Engineering, Osaka University, Suita, Osaka 565-0871, Japan \\ ${ }^{7}$ IPPLM Institute of Plasma Physics and Laser Microfusion, Warsaw, Poland \\ ${ }^{8}$ GSI Helmholtzzentrum für Schwerionenforschung GmbH-Planckstraß1, 64291 Darmstadt, Germany \\ ${ }^{9}$ Joint Institute for High Temperatures, RAS, 13 Bd.2 Izhorskaya st., Moscow 125412, Russia \\ ${ }^{10}$ Institute of Applied Physics, RAS, 46 Ulyanov Str., 603950 Nizhny Novgorod, Russia \\ ${ }^{11}$ National Research Nuclear University "MEPhI", 31 Kashirskoe shosse, Moscow 115409, Russia \\ Correspondence should be addressed to D. Mancelli; donaldi.mancelli@u-bordeaux.fr
}

Received 21 September 2021; Accepted 9 November 2021; Published 11 December 2021

Academic Editor: J. Manuel Perlado

Copyright (C) 2021 D. Mancelli et al. This is an open access article distributed under the Creative Commons Attribution License, which permits unrestricted use, distribution, and reproduction in any medium, provided the original work is properly cited.

\begin{abstract}
In this work, we present experimental results on the behavior of liquid water at megabar pressure. The experiment was performed using the HIPER (High-Intensity Plasma Experimental Research) laser facility, a uniaxial irradiation chamber of GEKKO XII (GXII) at the Institute of Laser Engineering (ILE), and the PHELIX at GSI (GSI Helmholtz Centre for Heavy Ion Research), a single-beam high-power laser facility, to launch a planar shock into solid multilayered water samples. Equation-of-state data of water $\left(\mathrm{H}_{2} \mathrm{O}\right)$ are obtained in the pressure range $0.50-4.6 \mathrm{Mbar}$ by tuning the laser-drive parameters. The Hugoniot parameters (pressure, density, etc.) and the shock temperature were simultaneously determined by using VISAR and SOP as diagnostic tools and quartz as the standard material for impedance mismatch experiments. Finally, our experimental results are compared with hydrodynamic simulations tested with different equations of state, showing good compatibility with tabulated SESAME tables for water.
\end{abstract}

\section{Introduction}

Equation of state (EOS) of matter at extreme thermodynamic states is of relevance for several fields in physics, especially in laboratory astrophysics and in inertial confinement fusion (ICF), where precise knowledge of the EOS is required. Water is predicted to be abundant in the outer planets of the Solar System, as well as in many of the recently discovered exoplanets. In particular, it is one of the principal ingredients of the mantles of giant planets such as Uranus, Neptune, and Jupiter [1]. The observation of large and asymmetric magnetic fields in these planets [2-4] indicated that the mantle is the origin of the field. As the dynamo theory requires the presence of a conductive material, it was 
suggested that one or all of the main ingredients of the mantle (ammonia, water, and methane, i.e., carbon $[5,6]$ ) experience a phase transition to a conducting state. Pioneering theoretical work has been done calculating the properties of the superionic phase of water at planetary conditions [7-9]. Therefore, water at very high pressures has been extensively studied in recent works [8, 10-14]. Recently $[15,16]$, the superionic phase of water was detected experimentally along the Uranus and Neptune isentropes at about $1.5 \mathrm{Mbar}$ by laser shocking water samples precompressed up to $28 \mathrm{kbar}$. The new superionic phase is predicted to span the pressure range of 1.5 to $6 \mathrm{Mbars}$.

Superionic water is a solid system that has high ionic conductivity well below the melting temperature. Whether $\mathrm{H}_{2} \mathrm{O}$ in planetary interiors is in the superionic or metallic state is of great importance for understanding the source of the planetary magnetic field.

Laser-driven shock compression is a useful technique for generating high pressure and temperature conditions similar to the inner part of Neptune and Uranus. However, measuring $P-\rho-T$ simultaneously is challenging, particularly temperature, as this requires absolute intensity measurements which are not necessary for the determination of the pressure or the density. In this work, we conducted laserdriven shock experiments on $\mathrm{H}_{2} \mathrm{O}$ samples up to $4.6 \mathrm{Mbar}$; along with pressure and density, we evaluated the temperature from measured reflectivity and thermal emission of the shocked sample.

The impedance mismatch (IM) method is widely used for the determination of Hugoniot of sample material. Given that one knows Hugoniot of a "standard" material that is used as a reference, the Rankine-Hugoniot (RH) [17] set of equations, expressing the conservation laws, can be used to relate the experimentally measured shock velocity $\left(D_{s}\right)$ in the standard material before the shock reaches the standardsample interface in the sample after it passes through the interface. In this work, we used $z$-cut $\alpha$-quartz $\left(\mathrm{SiO}_{2}\right)$ as a reference material $[18,19]$ which, at ambient pressure, is transparent to visible light and becomes reflective at pressures above 1 Mbar [20]. As diagnostic tools, we used the velocity interferometer system for any reflector (VISAR) [21-25] which allowed us to make a precise measurement of shock wave parameters and characterize the EOS of water. The RH set provides information of $P$ and $\rho$, but not of $T$, whereas $T$ is also an important thermodynamic parameter. Indeed, in this work, we also measured the temperature using streaked optical pyrometry (SOP) diagnostic data.

\section{Experimental Setup}

2.1. GEKKO XII-ILE Laser Facility. The first experiment was carried out on the HIPER (High-Intensity Plasma Experimental Research) laser facility, a uniaxial irradiation chamber of the GEKKO XII (GXII) laser at the Institute of Laser Engineering (ILE), Osaka University. Up to 12 beams of neodymium-doped yttrium aluminum garnet (Nd:YAG) laser, frequency tripled $(351 \mathrm{~nm})$, were used in the experiment. The laser pulse temporal profile was approximately square shape in time with full width at half maximum
(FWHM) $2.5 \mathrm{~ns}$ with typical rise and fall time of $100 \mathrm{ps}$ each. The focal spot diameter was $600 \mu \mathrm{m}$ flat top. SSD (smoothing by spectral dispersion technique) was applied to smooth out the beams. Kinoform phase plates were also used to achieve uniform irradiation, resulting in good shock front planarity.

Figure 1 shows the experimental setup and target configuration. A typical target assembly consisting of $10 \mu \mathrm{m}$ parylene $\left(\mathrm{C}_{8} \mathrm{H}_{8}\right)$ will be referred to as $\mathrm{CH} / 200 \mathrm{~nm} \mathrm{Au} / 100 \mu \mathrm{m}$ quartz (with AR@1054\&532 both sides) nominally, 500 $\mu \mathrm{m}$ water/100 $\mu \mathrm{m}$ quartz (with AR@1054\&532 both sides). The laser initially hits the $0.1 \mu \mathrm{m}$-thick $\mathrm{Al}$ layer, coated over the $\mathrm{CH}$ layer, to avoid laser shine through. The $\mathrm{CH}$ layer is the actual ablator, and quartz is the pusher layer. The $200 \mathrm{~nm}$ gold layer was placed to stop any X-rays from the plasma corona and avoid preheating of water. Water cells were produced at the Technical University of Darmstadt (Germany) target fabrication department. Due to the low $Z$ ablator and the low laser intensity, $\mathrm{X}$-ray radiation is low and characterized by low photon energy. The Au layer is capable to stop the X-ray radiation; thus, preheating of water is negligible.

The primary diagnostics were the VISAR and SOP. Two line-imaging interferometers (VISARs) allow to record time-resolved Doppler shift of the velocity of the fastmoving reflector and also the optical properties such as reflectivity [21-26]. These VISARs had different velocityper-fringe (VPF) sensitivities to resolve the $2 \pi$-phase jump ambiguities due to the shock velocity jump at material interfaces. The sensitivity of the two VISARs was $7.523 \mathrm{~km} / \mathrm{s}$ and $4.476 \mathrm{~km} / \mathrm{s}$ taking into account the refractive index of $\mathrm{SiO}_{2}$. The VISAR probe beam was an injection-seeded Qswitched YAG laser. The pulse duration of the probe was $\sim 10 \mathrm{~ns}$ at the FWHM, and its wavelength was $532 \mathrm{~nm}$. The postprocessing of the VISAR raw data obtained in the experiment allows determining [27] the fringe position to $10 \%$ of a fringe, while the multiple fringe shifts allow the precision of the shock velocity measurements to be a few percent. Our VISAR analysis showed uncertainties in measured $D_{s}$ of the order of $3 \%$.

To measure the shock temperature, we used SOP [28-30]. The self-emission of the shocked sample at wavelength $450 \mathrm{~nm}$ with $38 \mathrm{~nm}$ bandwidth was recorded spatially and temporally resolved, using an absolutely calibrated SOP.

2.2. PHELIX-GSI Laser Facility. The second experiment was conducted at the GSI facility using the PHELIX laser, a flashlamp-pumped Nd:glass laser utilizing the second harmonic at wavelength $527 \mathrm{~nm}$. The spatial profile was flat top with spot $350 \mu \mathrm{m}$ FWHM obtained by an appropriate phase plate [31], and the temporal profile was top hat with a duration of $3.5 \mathrm{~ns}$ (FWHM). The laser was focused onto a multilayered target with intensity $I 5.49 \times 10^{12} \mathrm{~W} / \mathrm{cm}^{2}$ to $2.52 \times 10^{13} \mathrm{~W} /$ $\mathrm{cm}^{2}$. The ablation pressure was approximately $12 \mathrm{Mbar}$, was generated in our plastic ablator (parylene with gross chemical formula $\mathrm{C}_{8} \mathrm{H}_{8}$ ), and was estimated from the wellknown scaling laws [32-34] for given intensities.

$$
P=8.6\left(\frac{I}{10^{14}}\right)^{2 / 3} \lambda^{-2 / 3}\left(\frac{A}{2 Z}\right)^{1 / 3}
$$




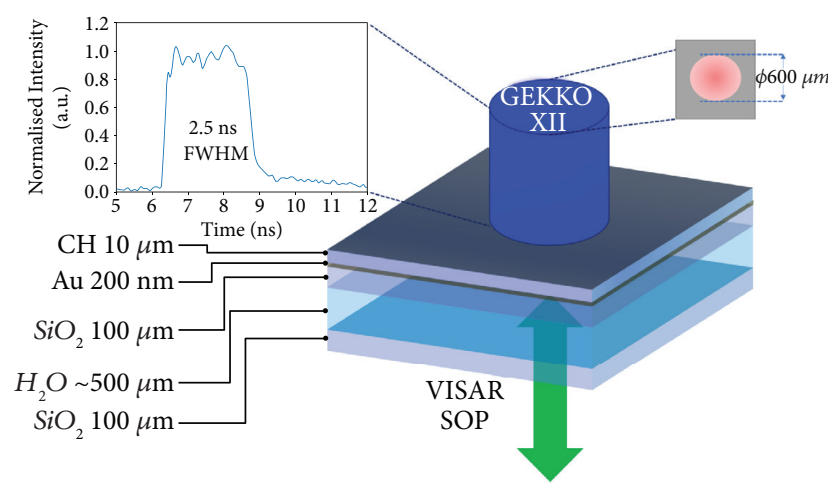

Figure 1: Experimental setup: sketch of the multilayered target design in planar geometry with its associated thicknesses used in our experiment. Drive laser from top. From bottom, the VISAR and SOP measure the shock velocity and the self-emission of the shocked sample on the rear side.

where $A$ and $Z$ are the atomic mass number and the atomic number of the target material: $I$ is in $\mathrm{W} / \mathrm{cm}^{2}, P$ in Mbar, and $\lambda$ in $\mu \mathrm{m}$. A schematic of the PHELIX experimental setup is shown in Figure 2. Targets were also produced at the Technical University of Darmstadt (Germany) target fabrication department.

The target configuration and the experimental setup are presented in Figure 2. The sample consists of $15 \mu \mathrm{m} \mathrm{C} \mathrm{CH}_{8}$ (parylene) $/ 5 \mu \mathrm{m}$ epoxy/ $10 \mu \mathrm{m} \mathrm{Al} / 7 \mu \mathrm{m}$ epoxy/100 $\mu \mathrm{m}$ quartz (with AR@1054\&527 both sides) nominally, 500 $\mu \mathrm{m}$ water/ $100 \mu \mathrm{m}$ quartz (with AR@1054\&527 both sides). The laser initially hits the $0.1 \mu \mathrm{m}$-thick Al layer, coated over the $\mathrm{CH}$ layer, to avoid direct laser shine through. Epoxy was used to glue $\mathrm{CH} / \mathrm{Al}$ and $\mathrm{Al} /$ quartz interfaces. The $\mathrm{CH}$ layer is the actual ablation layer, and quartz is the pusher layer. The $10 \mu \mathrm{m} \mathrm{Al} \mathrm{layer} \mathrm{was} \mathrm{placed} \mathrm{to} \mathrm{stop} \mathrm{any} \mathrm{X-rays} \mathrm{from} \mathrm{the}$ plasma corona and avoid preheating of the quartz/water layers. The VISAR laser had a wavelength of $660 \mathrm{~nm}$, and the associated sensitivities were $1.285 \mathrm{~km} / \mathrm{s} / \mathrm{f}$ and $4.7 \mathrm{~km} / \mathrm{s} / \mathrm{f}$, respectively. Also, SOP was looking at the self-emission of the shocked target; however, here, we report only VISAR results and compare with findings on water samples from the GEKKO XII laser facility.

\section{Impedance Mismatch Method: Single- Shock Data}

The impedance-matching method $[5,17-19,35]$ was used to estimate the shock state in water after passing the quartz/ water interface; an illustrative method is shown in Figure 3. Because of the impedance mismatch at the $\mathrm{SiO}_{2} / \mathrm{H}_{2} \mathrm{O}$ interface, the shock wave produced a transmitted shock into $\mathrm{H}_{2} \mathrm{O}$ and a reflected rarefaction wave propagating back into quartz. In the rarefaction wave, the shock-compressed quartz undergoes isentropic release until its pressure and particle velocity match those of shocked water. The IM method requires precise knowledge of Hugoniot and release behavior of the standard reference material (quartz in our case) and the Rankine-Hugoniot (RH) jump relations, which are derived from the conservation laws, mass,

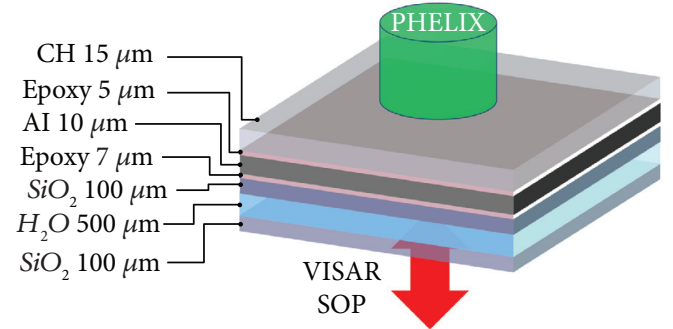

FIGURE 2: Experimental setup: a sketch of the multilayered target design at the PHELIX laser facility in planar geometry with its associated thicknesses used in our experiment. Drive laser from the top. Bottom: the VISAR and SOP diagnostics which measure the shock velocity and the self-emission of the shocked target on the rear side.

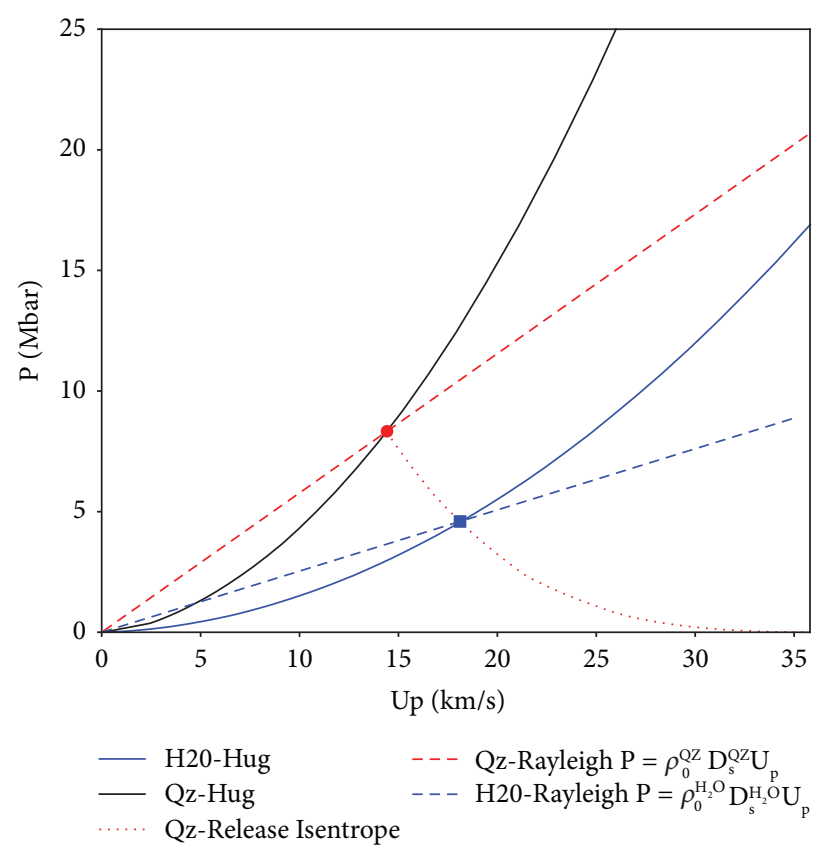

Figure 3: $P-U_{p}$ graphical construction of the impedance mismatch procedure for the highest pressure shot of the quartz standard. The shocked state of $\mathrm{H}_{2} \mathrm{O}$ is constrained to remain on the incident Rayleigh line (blue dashed line) defined for $D_{s}^{Q z}=21.8(\mathrm{~km} / \mathrm{s})$ for this case. The solid red and dashed lines are the Hugoniot and the Rayleigh line of $\left(\mathrm{SiO}_{2}\right)$, respectively, associated with the release curve calculated for $D_{s}^{Q z}=21.8(\mathrm{~km} / \mathrm{s})$. The crossing of the Rayleigh line with the release isentrope path (blue square) determines the IM point and provides the experimental value of $\left(P^{\mathrm{H}_{2} \mathrm{O}}, U_{p}^{\mathrm{H}_{2} \mathrm{O}}\right)$ which may or may not lay on the shock polar for water, i.e., may (or may not) be in agreement with the used theoretical EOS.

momentum, and energy to close the system and derive all the remaining thermodynamic parameters:where $\rho, D_{s}, U_{p}, P$, and $\varepsilon$ denote the density, shock velocity, particle velocity, pressure, and internal energy behind the shock. The initial states are denoted with subscript 0 . The third equation plotted in the $(P-U)$ plane gives the so-called Rayleigh line of the material. Figure 3 illustrates such a method, deriving the shock pressure in water and the particle velocity. The 
measurement of $D_{s}^{Q z}$ gives the shock state $\left(P^{Q z}, U_{p}^{Q z}\right)$. The crossing of the isentropic release of the quartz standard with the Rayleigh line of the water sample determines the shock state in water $\left(P^{\mathrm{H}_{2} \mathrm{O}}, U_{p}^{\mathrm{H}_{2} \mathrm{O}}\right)$.

$$
\begin{aligned}
\frac{\rho}{\rho_{0}} & =\frac{D_{s}}{D_{s}-U_{p}}, \\
P-P_{0} & =\rho_{0} D_{s} U_{p}, \\
\varepsilon-\varepsilon_{0} & =\frac{1}{2}\left(P+P_{0}\right)\left(\frac{1}{\rho_{0}}-\frac{1}{\rho}\right) .
\end{aligned}
$$

\section{Results and Discussion}

Figure 4 presents the raw data obtained with the VISAR for the shot SID-43058. The arrival time of the shock at the Au/ $\mathrm{SiO}_{2}$ interface front side defines the time zero $\left(t_{0}=0\right)$. Then, while the shock front enters into $\mathrm{SiO}_{2}$, it becomes highly reflective, and its velocity gradually decreases. It is indicated in the raw image with a dotted line as $t_{0}$. At $t_{1}=7.21 \mathrm{~ns}$, the shock front strikes the $\mathrm{H}_{2} \mathrm{O}$ layer as shown in Figure 4. The velocity profile and the reflectivity obtained from Figure 4 are shown in Figure 5. The transmitted shock in $\mathrm{H}_{2} \mathrm{O}$ also becomes reflective. The shock velocity in $\mathrm{SiO}_{2}$, used in the impedance mismatch analysis, was obtained by analysing VISAR images first by using shock chronometry, i.e., the average shock velocity in $\mathrm{SiO}_{2}$ was given by $D_{s}^{Q z}=100 \mu \mathrm{m} /\left(t_{1}-t_{0}\right)$. Then, the analysis is refined by looking at the displacement of the fringe shift and averaging the shock velocities from the two VISAR records taking into account the temporal resolution of the streak camera and the sensitivities of each VISAR. The IM method is applied using the instantaneous value of the shock velocity just before $t_{1}$.

To determine $D_{s}^{\mathrm{H}_{2} \mathrm{O}}$, we instead could rely only on the VISAR images. The experimental results were supported with numerical simulation utilizing MULTI-1D, which is a one-dimensional radiation hydrodynamic code [36]. Simulations show that, in the present experiment, $D_{s}^{Q z}$ decays. We observe a decay in the case of $D_{s}^{\mathrm{H}_{2} \mathrm{O}}$. Detailed interpretation of simulations will be shown in Section 6 .

Getting Hugoniot points for $\mathrm{H}_{2} \mathrm{O}$ requires that one knows the release isentrope (RI) of $\mathrm{SiO}_{2}$ from its shocked state. This study uses an EOS model to calculate the release isentrope for each shock state in $\mathrm{SiO}_{2}$ utilizing an in-house Python script. The IM method provides the Hugoniot state of $\mathrm{H}_{2} \mathrm{O}$ as the locus where the release isentrope of $\mathrm{SiO}_{2}$ crosses the Rayleigh line $\left(P=\rho_{0}^{\mathrm{H}_{2} \mathrm{O}} D_{s}^{\mathrm{H}_{2} \mathrm{O}} U_{p}\right)$ of water.

Such a method is shown in Figure 3, with the Hugoniot and the RI of $\mathrm{SiO}_{2}$ (black line and red dotted curve, respectively). Dashed lines denote the Rayleigh lines of $\mathrm{SiO}_{2}$ and water (red and blue), respectively, as well as their associated Hugoniot. The Hugoniot curve is derived from the SESAME database [37] and the QEOS [38], tabulated data 7385 for quartz and 7154 for water. The obtained shocked states of water are compared with other available works and
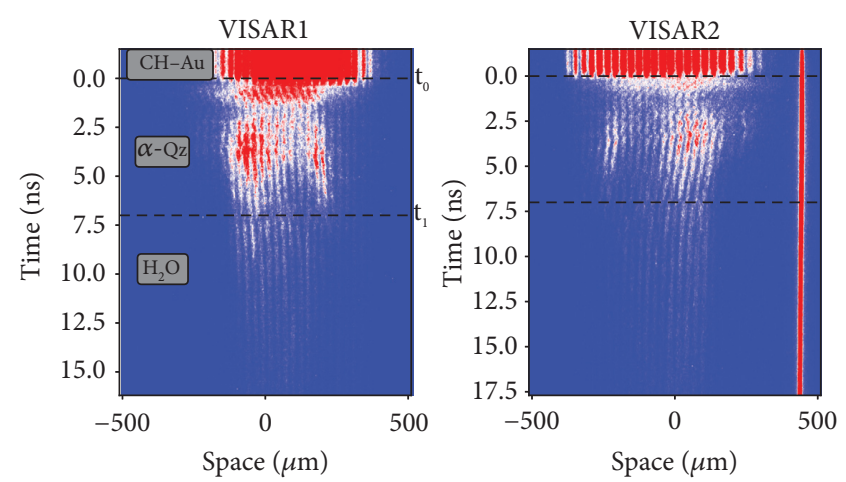

FIGURE 4: Experimentally obtained VISAR images for the shot SID43058 .


FIgURE 5: Top: evolution of the shock velocity in time for $\mathrm{SiO}_{2}$ and $\mathrm{H}_{2} \mathrm{O}$ extracted from Figure 4: VISAR-1 (black) and VISAR-2 (yellow). Bottom: the reflectivity vs. time. This shows an increase after the shock enters the $\mathrm{SiO}_{2}$ layer owing to the reverberating wave in $\mathrm{SiO}_{2}$ (at about $t \approx 3-4 \mathrm{~ns}$ ), and a second increase appears when the shock passes the $\mathrm{SiO}_{2}-\mathrm{H}_{2} \mathrm{O}$ interface at time $t_{1}$. Shock velocities are listed in Table 1 (SID-43058).

are shown in Figure 6 in the $P-\rho$ plane and in Figure 7 in the $P-U_{p}$ plane.

\section{Temperature Measurement}

The shock front emits thermal radiation, and which, in our experiment, was collected by the SOP $[28,44]$. The absolute spectral radiance of the shock front was measured at a wavelength of $450 \mathrm{~nm}$ with bandwidth $38 \mathrm{~nm}$. Assuming a grey body spectrum, the spectral radiance I $(450 \mathrm{~nm})$ is given by Planck's black body emissivity corrected for the absorptivity of the material $(\varepsilon=1-R)$ calculated using the reflectivity $R$ of the shock front measured by VISARs since the light is either absorbed or reflected. Then, 
TABLE 1: Hugoniot data from the impedance mismatch method with $\alpha$-quartz as a reference material. The velocity of shock in quartz $D^{\mathrm{Q} z}$ and water $D^{\mathrm{H}_{2} \mathrm{O}}$ was used in the IM analysis to obtain the particle velocity $U_{p}^{\mathrm{H}_{2} \mathrm{O}}$, pressure $P_{\mathrm{H}_{2} \mathrm{O}}$, and density $\rho_{\mathrm{H}_{2} \mathrm{O}}$ on water Hugoniot. The compressibility $\left(\rho / \rho_{0}\right)^{\mathrm{H}_{2} \mathrm{O}}$ was calculated by dividing $\rho^{\mathrm{H}_{2} \mathrm{O}}$ by the initial density. Water target was studied at two different high-power laser facilities.

\begin{tabular}{|c|c|c|c|c|c|c|c|c|c|c|}
\hline Facility & $\begin{array}{l}\text { Shot } \\
\text { ID }\end{array}$ & $\begin{array}{c}\rho_{0}^{\mathrm{Qz}} \\
\left(\mathrm{g} / \mathrm{cm}^{3}\right)\end{array}$ & $\begin{array}{c}\rho_{0}^{\mathrm{H}_{2} \mathrm{O}} \\
\left(\mathrm{g} / \mathrm{cm}^{3}\right)\end{array}$ & $\begin{array}{c}D^{Q z} \\
(\mathrm{~km} / \mathrm{s})\end{array}$ & $\begin{array}{l}D^{\mathrm{H}_{2} \mathrm{O}} \\
(\mathrm{km} / \mathrm{s})\end{array}$ & $\begin{array}{l}U_{p}^{\mathrm{H}_{2} \mathrm{O}} \\
(\mathrm{km} / \mathrm{s})\end{array}$ & $\begin{array}{l}P^{\mathrm{H}_{2} \mathrm{O}} \\
(\mathrm{Mbar})\end{array}$ & $\varepsilon-\varepsilon_{0}(\mathrm{~kJ} / g)$ & $\begin{array}{c}\rho^{\mathrm{H}_{2} \mathrm{O}} \\
\left(\mathrm{g} / \mathrm{cm}^{3}\right)\end{array}$ & $\rho / \rho_{0}$ \\
\hline \multirow{4}{*}{$\begin{array}{l}\text { GEKKO XII- } \\
\text { ILE }\end{array}$} & 43045 & 2.65 & 0.9848 & $21.8 \pm 0.3$ & $25.7 \pm 0.4$ & $18.2 \pm 0.2$ & $4.6 \pm 0.2$ & $164.4 \pm 10.6$ & $3.3 \pm 0.1$ & 3.4 \\
\hline & 43051 & 2.65 & 0.9848 & $16.1 \pm 0.2$ & $18.6 \pm 0.2$ & $12.5 \pm 0.1$ & $2.3 \pm 0.09$ & $78.8 \pm 5.1$ & $3.00 \pm 0.1$ & 3.0 \\
\hline & 43058 & 2.65 & 0.9848 & $14.0 \pm 0.1$ & $16.0 \pm 0.2$ & $10.4 \pm 0.1$ & $1.6 \pm 0.06$ & $54.7 \pm 3.3$ & $2.8 \pm 0.10$ & 2.8 \\
\hline & 43063 & 2.65 & 0.9848 & $10.0 \pm 0.1$ & $11.6 \pm 0.1$ & $7.0 \pm 0.1$ & $0.8 \pm 0.02$ & $20.9 \pm 1.3$ & $2.5 \pm 0.07$ & 2.5 \\
\hline \multirow{4}{*}{ PHELIX GSI } & S20 & 2.65 & 0.9848 & $8.9 \pm 0.1$ & $9.8 \pm 0.1$ & $5.4 \pm 0.1$ & $0.5 \pm 0.01$ & $14.7 \pm 0.9$ & $2.1 \pm 0.05$ & 2.2 \\
\hline & S21 & 2.65 & 0.9848 & $18.9 \pm 0.2$ & $22.1 \pm 0.3$ & $15.5 \pm 0.2$ & $3.4 \pm 0.1$ & $120.9 \pm 7.8$ & $3.3 \pm 0.16$ & 3.3 \\
\hline & S27 & 2.65 & 0.9848 & $12.9 \pm 0.1$ & $14.5 \pm 0.2$ & $9.3 \pm 0.1$ & $1.3 \pm 0.05$ & $43.3 \pm 2.8$ & $2.7 \pm 0.1$ & 2.8 \\
\hline & S32 & 2.65 & 0.9848 & $10.8 \pm 0.1$ & $12.0 \pm 0.1$ & $7.2 \pm 0.1$ & $0.87 \pm 0.03$ & $26.2 \pm 1.7$ & $2.4 \pm 0.07$ & 2.5 \\
\hline
\end{tabular}



FIgURE 6: Water Hugoniot data in the $P-\rho$ plane. Squared data points: our experimental points. The lines correspond to tabulated data from SESAME (SESAME 7150, 7153, and 7154 and $P_{0}=10 \mathrm{kbar}$ ) [37]. Density functional theory-molecular dynamics (DFT-MD) calculations of Hugoniot of water form French et al. [9, 39].

$$
I(\lambda, T)=A(\lambda) \frac{2 h c^{2}}{\lambda^{5}} \frac{1}{e^{h c / \lambda k_{B} T}-1} .
$$

The temperature was determined using this formula (where $h, c$, and $k_{B}$ are Planck's constant, speed of light, and Boltzmann's constants, respectively). $A(\lambda)$ is a parameter taking into account the reflectivity, the sensitivity of the streak, the geometry, and the collection optics path.

Solving equation (5) with respect to temperature, we get

$$
T=\frac{h c}{\lambda k_{B} \ln \left(\left(2 h c^{2} A(\lambda) / I(\lambda) \lambda^{5}\right)+1\right)} .
$$




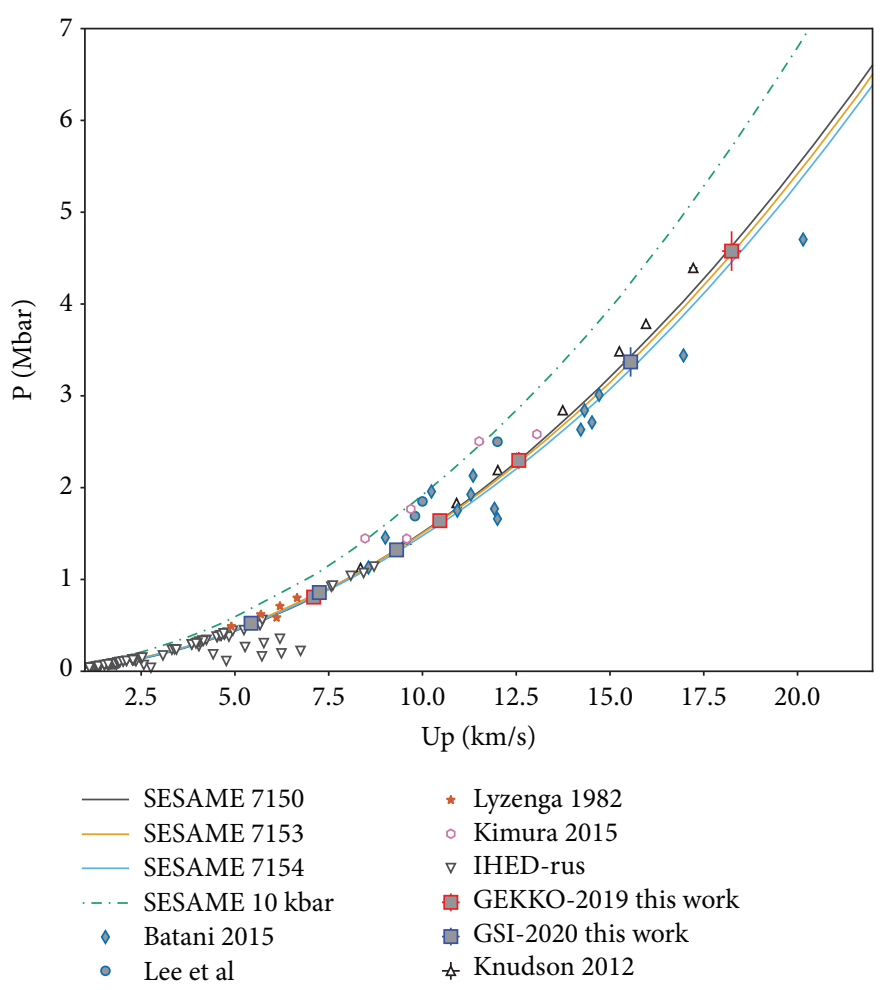

FIgURE 7: Water Hugoniot data in the $P-U_{p}$ plane. Our data showed good agreement with the SESAME models available for water.

SOP calibration has been made in situ using the quartz standard (GEKKO XII) [42, 45]. For the temperature estimation, we used the mean reflectivity of the two VISAR records which were taken simultaneously for each shot. The results of SOP temperature estimation are shown in Figure 8 where we report two shots at different laser energies with the associated temperature profile in time (Figure 8(a)).

The temperature results as a function of pressure reaching the water interface are shown in Figure 9 together with results from Lyzenga et al. [41], Kimura et al. [10], and Guarguaglini et al. [42] and Hugoniot tabulated curves based on SESAME models (SESAME 7150, 7153, and 7154 and $P_{0}=10 \mathrm{kbar}$ ) and ab initio Hugoniot from French et al. [39].

\section{Hydro Simulations}

$1 \mathrm{D}$ radiative hydrodynamic simulations were performed with MULTI-1D [36] to comprehend our experimental results. The laser temporal profile of the pulse was flat top in time with a plateau duration of $2.5 \mathrm{~ns}$ at FWHM and rise and fall times of $0.1 \mathrm{~ns}$. In the simulation, we utilized the SESAME tables of the following materials which consist of our target SESAME table 7770 for parylene [37], SESAME table 2700 for gold [37], SESAME 7385 for quartz [37], and SESAME tables 7150, 7153, and 7154 for water [37]. Concerning the water EOS, we compared different models of EOS tables coming from the SESAME database [37], from QEOS [38], and from FEOS [46], in all cases setting the initial density at $\rho_{0}=0.98\left(\mathrm{~g} / \mathrm{cm}^{3}\right)$ in order to check the validity of the EOS data. The density and pressure map, reproducing the shot SID-43058 obtained from 1D simulation, is shown in Figures 10(a) and 10(b), respectively. Figure 10(c) shows the change of the maximum density postprocessed from Figure 10(a) at interval times, and Figure 10(d) shows the change of pressure along the shock front of the simulated target top (Figure 10(b)), respectively. The use of $1 \mathrm{D}$ simulation to interpret the experimental results is confirmed because of the use of appropriate phase plate resulting in a large focal spot $(\sim 600 \mu \mathrm{m})$. In addition to this, the justification of the $1 \mathrm{D}$ approximation is supported by two experimental observations; such results can be seen in Figure 4 where good planarity of the shock is observed.

Raw data from VISAR images (see Figure 4) indicate that the shock breakout is quite flat both at metal/ $\mathrm{SiO}_{2}$ and at $\mathrm{SiO}_{2} / \mathrm{H}_{2} \mathrm{O}$ interfaces. At this point, we must note that $2 \mathrm{D}$ effects will result in curvature, initially affecting the edges of the shock front, yet gradually advancing to the center. However, in our experimental results, the curvature of the shock front is not observed suggesting that the $2 \mathrm{D}$ effects in hydrodynamics can be neglected. In our case, the velocity of the shock is maintained and decaying quite slowly, and actually (within error bars), the decay obtained by analysing the VISAR images is compatible with the results of $1 \mathrm{D}$ simulations. The reduction of shock pressure and velocity during propagation is caused by two phenomena: (a) the release wave from the target front side catching up the traveling shock and (b) 2D effects in shock front propagation. In fact, due to the fair agreement of conducted experiment with 1D simulations, we conclude that (b) is not important. Indeed, a much faster 


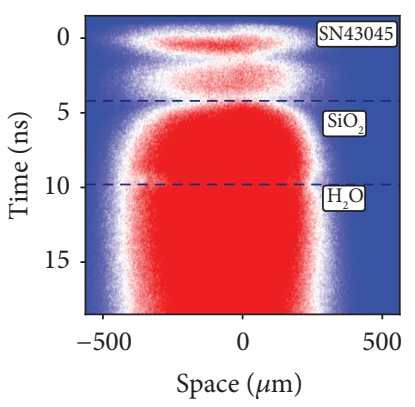

(a)

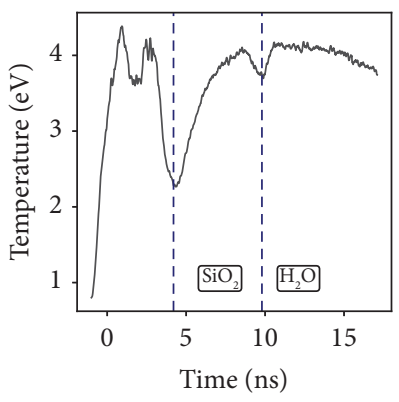

(b)

FIGURE 8: (a) SOP result for shot SN43045 at a laser energy of $1.1 \mathrm{~kJ}$, the highest energy shot obtained in our experiment, and (b) temperature estimation taking into account the mean reflectivity of VISAR-1 and VISAR-2. The initial luminosity ( $t \leq 4$ ns) is due to scattered laser light.

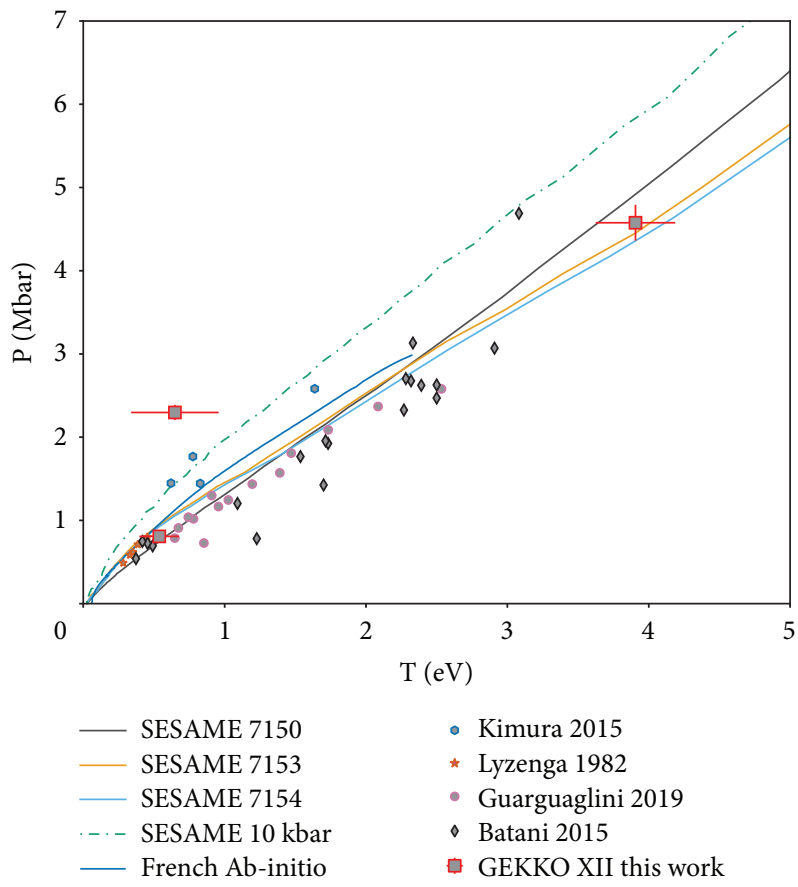

FIgURE 9: Principal Hugoniot data in the $P-T$ plane obtained in this study from the GEKKO XII-ILE laser facility, compared with Lyzenga et al. [41], Kimura et al. [10], Guarguaglini et al. [42], and Batani et al. [43]. Also shown are SESAME models for three different tabulated EOS data at slightly different initial densities.

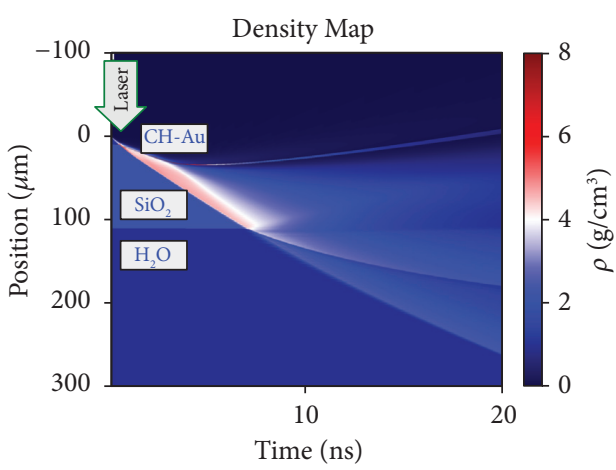

(a)

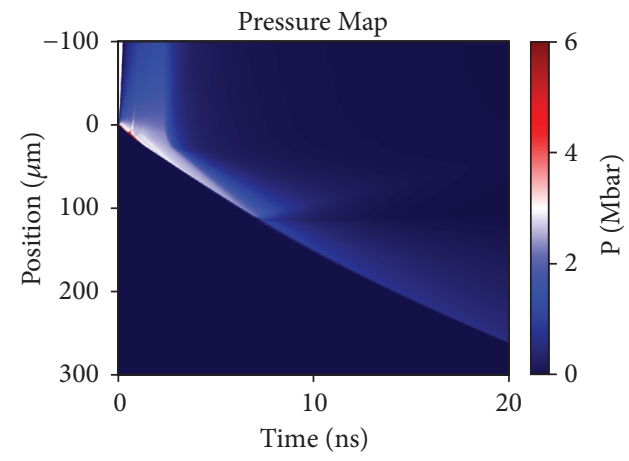

(b)

Figure 10: Continued. 


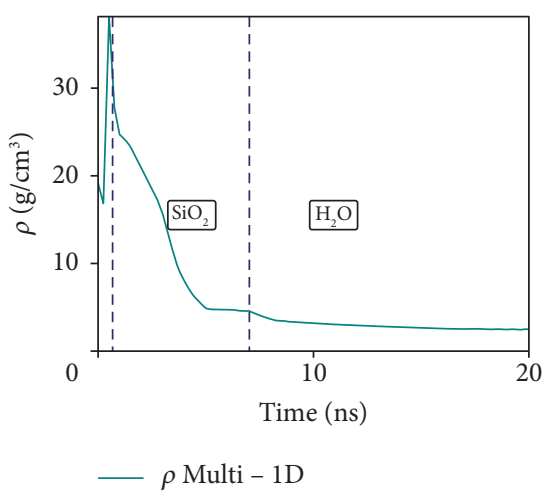

(c)

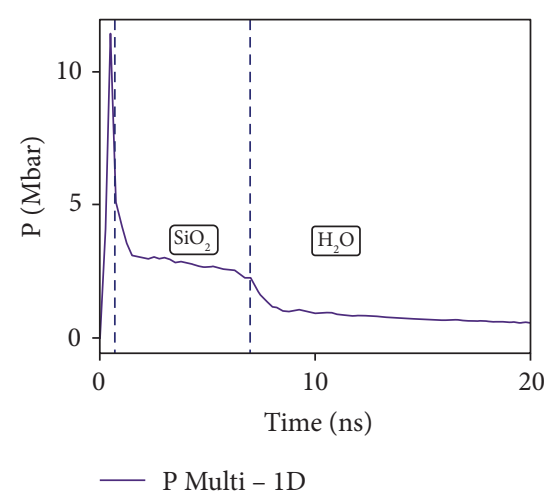

(d)

Figure 10: Laser drive beam hits the target from the top as indicated by the arrow symbol; the constituent layers are also indicated with the right order $\left(\mathrm{CH} / \mathrm{Au} / \mathrm{SiO}_{2}\right.$ /water). (a) Hydrodynamic progression of the density map obtained from MULTI-1D reproducing the experimental results of shot SID-43058 with nominal laser intensity $1.4 \times 10^{13} \mathrm{~W} / \mathrm{cm}^{2}$; for this particular shot, 3 beams/12 were used for the main drive delivering a total of $252 \mathrm{~J}$ on the target. With the above laser parameter, the simulation reproduces well the shock breakout time in the $\mathrm{SiO}_{2}-\mathrm{H}_{2} \mathrm{O}$ inner interface. (b) Variation of the pressure map for the same shot conditions. (c) Time evolution of pressure and density forms MULTI simulation for shot SID-43058. (d) Change of pressure at the shock front up to the maximum simulation time.

decay of shock pressure and velocity would be expected if $2 \mathrm{D}$ effects were important.

\section{Conclusion}

In summary, we obtained EOS data of water along the principal Hugoniot up to $5 \mathrm{Mbar}$. Water samples, contained within a multilayered water cell, were dynamically compressed in planar geometry using the high-power laser facilities GEKKO XII (ILE) and PHELIX (GSI). Utilizing quartz as a standard material in both experimental campaigns and the main diagnostics such as VISAR/SOP substantially reduced experimental errors in optimized experimental conditions. The impedance mismatching analysis allowed to verify that $P, \rho,\left(\varepsilon-\varepsilon_{0}\right)$, and the Hugoniot data are in fair agreement with those predicted by SESAME table 7150, and on the contrary, they show a significant difference concerning the Hugoniot curve calculated using DFT-MD simulations. Also, our experimental outcome showed good agreement with simulations performed with the radiative hydro code MULTI-1D using the SESAME tabulated EOS, the QEOS model, and the FEOS model, a modified version of the QEOS. The agreement with 1D simulations shows indeed that, in our experimental setup, 2D effects in hydrodynamics are negligible, a result which mainly depends on the use of laser focal spots. For a few shots, we could also measure the temperature of shocked material using calibrated SOP diagnostics. Our data confirm previous experimental results and show that, in the pressure range up to $4.6 \mathrm{Mbar}$, water is in a reflective state.

\section{Data Availability}

The data that support the findings of this study are available from the corresponding author upon reasonable request.

\section{Disclosure}

This work has been carried out within the framework of the EUROfusion Consortium. The views and opinions expressed herein do not necessarily reflect those of the European Commission. Our findings were presented at the Satellite Meeting of the 47th European Conference on Plasma Physics.

\section{Conflicts of Interest}

The authors declare that they have no conflicts of interest.

\section{Acknowledgments}

The authors would like to acknowledge the support of the laser technical team at GEKKO XII (ILE) and PHELIX (GSI). This work received funding from the Euratom Research and Training Program 2014-2018 and 2019-2020 (Grant agreement no. 633053). The involved teams were operated within the framework of the Enabling Research Project ENRIFE19.CEA-01, Study of Direct Drive and Shock Ignition for IFE: Theory, Simulations, Experiments, Diagnostics Development. JIHT RAS team members were supported by the Ministry of Science and Higher Education of the Russian Federation (State Assignment no. 075-00460-21-00).

\section{References}

[1] S. Mazevet, A. Licari, G. Chabrier, and A. Y. Potekhin, “Ab initio based equation of state of dense water for planetary and exoplanetary modeling," Astronomy \& Astrophysics, vol. 621, p. A128, 2019.

[2] N. F. Ness, M. H. Acuña, K. W. Behannon et al., "Magnetic fields at Uranus," Science, vol. 233, no. 4759, pp. 85-89, 1986.

[3] J. E. P. Connerney, M. H. Acuña, and N. F. Ness, "The magnetic field of Neptune," Advances in Space Research, vol. 12, no. 8, pp. 239-248, 1992. 
[4] Z. Chen, X. Na, C. B. Curry et al., "Observation of a highly conductive warm dense state of water with ultrafast pumpprobe free-electron-laser measurements," Matter and Radiation at Extremes, vol. 6, no. 5, Article ID 054401, 2021.

[5] P. Wang, C. Zhang, S. Jiang et al., "Density-dependent shock hugoniot of polycrystalline diamond at pressures relevant to ICF," Matter and Radiation at Extremes, vol. 6, no. 3, Article ID 035902, 2021.

[6] K. Jakubowska, D. Mancelli, R. Benocci et al., "Reflecting laser-driven shocks in diamond in the megabar pressure range," High Power Laser Science and Engineering, vol. 9, no. e3, 2021.

[7] C. Cavazzoni, G. L. Chiarotti, S. Scandolo, E. Tosatti, M. Bernasconi, and M. Parrinello, "Superionic and metallic states of water and ammonia at giant planet conditions," Science, vol. 283, no. 5398, pp. 44-46, 1999.

[8] T. R. Mattsson and M. P. Desjarlais, "Phase diagram and electrical conductivity of high energy-density water from density functional theory," Physical Review Letters, vol. 97, Article ID 017801, 2006.

[9] M. French, T. R. Mattsson, N. Nettelmann, and R. Redmer, "Equation of state and phase diagram of water at ultrahigh pressures as in planetary interiors," Physical Review B: Condensed Matter, vol. 79, Article ID 054107, 2009.

[10] T. Kimura, N. Ozaki, T. Sano et al., "P- $\rho$-T measurements of $\mathrm{H}_{2} \mathrm{O}$ up to $260 \mathrm{GPa}$ under laser-driven shock loading," The Journal of Chemical Physics, vol. 142, no. 16, Article ID 164504, 2015.

[11] N. Goldman, E. J. Reed, I.-F. W. Kuo, L. E. Fried, C. J. Mundy, and A. Curioni, "Ab initio simulation of the equation of state and kinetics of shocked water," The Journal of Chemical Physics, vol. 130, no. 12, Article ID 124517, 2009.

[12] D. Pan, Q. Wan, and G. Galli, "The refractive index and electronic gap of water and ice increase with increasing pressure," Nature Communications, vol. 5, no. 1, p. 3919, 2014.

[13] D. H. Dolan, M. D. Knudson, C. A. Hall, and C. Deeney, "A metastable limit for compressed liquid water," Nature Physics, vol. 3, no. 5, pp. 339-342, 2007.

[14] K. Nagayama, Y. Mori, K. Shimada, and M. Nakahara, "Shock hugoniot compression curve for water up to $1 \mathrm{GPa}$ by using a compressed gas gun," Journal of Applied Physics, vol. 91, no. 1, pp. 476-482, 2002.

[15] M. Millot, S. Hamel, J. R. Rygg et al., "Experimental evidence for superionic water ice using shock compression," Nature Physics, vol. 14, no. 3, pp. 297-302, 2018.

[16] M. Millot, F. Coppari, J. R. Rygg et al., "Nanosecond X-ray diffraction of shock-compressed superionic water ice," $\mathrm{Na}$ ture, vol. 569, no. 7755, pp. 251-255, 2019.

[17] I. A. B. Zel'dovich and Y. P. Raizer, "Physics of shock waves and high-temperature hydrodynamic phenomena," Dover Books on Physics, Dover Publications, Mineola, NY, USA, 2002.

[18] M. D. Knudson and M. P. Desjarlais, "Adiabatic release measurements in $\alpha$-quartz between 300 and $1200 \mathrm{GPa}$ characterization of $\alpha$-quartz as a shock standard in the multimegabar regime," Physical Review B: Condensed Matter, vol. 88, Article ID 184107, 2013.

[19] S. Brygoo, M. Millot, P. Loubeyre et al., "Analysis of laser shock experiments on precompressed samples using a quartz reference and application to warm dense hydrogen and helium," Journal of Applied Physics, vol. 118, no. 19, Article ID 195901, 2015.

[20] Y. Laudernet, J. Clérouin, and S. Mazevet, "Ab initiosimulations of the electrical and optical properties of shock-compressed $\mathrm{SiO}_{2}$," Physical Review B, vol. 70, no. 16, Article ID 165108, 2004.

[21] P. M. Celliers, D. K. Bradley, G. W. Collins, D. G. Hicks, T. R. Boehly, and W. J. Armstrong, "Line-imaging velocimeter for shock diagnostics at the omega laser facility," Review of Scientific Instruments, vol. 75, no. 11, pp. 4916-4929, 2004.

[22] D. D. Bloomquist and S. A. Sheffield, "Optically recording interferometer for velocity measurements with subnanosecond resolution," Journal of Applied Physics, vol. 54, no. 4, pp. 1717-1722, 1983.

[23] C. A. Bolme and K. J. Ramos, "Line-imaging velocimetry for observing spatially heterogeneous mechanical and chemical responses in plastic bonded explosives during impact," Review of Scientific Instruments, vol. 84, no. 8, Article ID 083903, 2013.

[24] L. M. Barker and R. E. Hollenbach, "Laser interferometer for measuring high velocities of any reflecting surface," Journal of Applied Physics, vol. 43, no. 11, pp. 4669-4675, 1972.

[25] L. M. Barker and K. W. Schuler, "Correction to the velocityper-fringe relationship for the VISAR interferometer," Journal of Applied Physics, vol. 45, no. 8, pp. 3692-3693, 1974.

[26] Z. Yan, H. Liu, X. Zhang et al., "Dynamics of particles near the surface of a medium under ultra-strong shocks," Matter and Radiation at Extremes, vol. 6, no. 2, Article ID 026903, 2021.

[27] V. T. F. A. Neutrino, "A light, expandable and full featured image analysis tool for research,” 2011, https://github.com/ NeutrinoToolkit/Neutrino.

[28] J. E. Miller, T. R. Boehly, A. Melchior et al., "Streaked optical pyrometer system for laser-driven shock-wave experiments on omega," Review of Scientific Instruments, vol. 78, no. 3, Article ID 034903, 2007.

[29] M. C. Gregor, R. Boni, A. Sorce et al., "Absolute calibration of the omega streaked optical pyrometer for temperature measurements of compressed materials," Review of Scientific Instruments, vol. 87, no. 11, Article ID 114903, 2016.

[30] Z. He, G. Jia, F. Zhang et al., "Calibration and verification of streaked optical pyrometer system used for laser-induced shock experiments," High Power Laser Science and Engineering, vol. 7, Article ID e49, 2019.

[31] M. Koenig, B. Faral, J. M. Boudenne, D. Batani, A. Benuzzi, and S. Bossi, "Optical smoothing techniques for shock wave generation in laser-produced plasmas," Physical Review E, vol. 50, no. 5, pp. R3314-R3317, 1994.

[32] P. Mora, "Theoretical model of absorption of laser light by a plasma," Physics of Fluids, vol. 25, no. 6, pp. 1051-1056, 1982.

[33] J. Lindl, "Development of the indirect-drive approach to inertial confinement fusion and the target physics basis for ignition and gain," Physics of Plasmas, vol. 2, no. 11, pp. 3933-4024, 1995.

[34] P. Chen, R. Hu, H. Zhou et al., "Numerical investigation of radiation ablation and acceleration of high-density carbon foils," Laser and Particle Beams, vol. 38, no. 4, pp. 239-243, 2020.

[35] M. C. Marshall, A. E. Lazicki, D. Erskine et al., "Developing quartz and molybdenum as impedance-matching standards in the 100-mbar regime," Physical Review B, vol. 99, no. 17, Article ID 174101, 2019.

[36] R. Ramis, R. Schmalz, and J. Meyer-Ter-Vehn, "Multi-a computer code for one-dimensional multigroup radiation hydrodynamics," Computer Physics Communications, vol. 49, no. 3, pp. 475-505, 1988.

[37] T4 Group LANL, "Eos sesame tables developed at the Los Alamos laboratory (sesame report on the los alamos equation-ofstate library, report no. lalp-83-4, 1983, and report la-ur-92-3407, 
1992, t4 group lanl, Los Alamos),” Report LA-UR-92-3407, 1992.

[38] R. M. More, K. H. Warren, D. A. Young, and G. B. Zimmerman, "A new quotidian equation of state (QEOS) for hot dense matter," Physics of Fluids, vol. 31, no. 10, pp. 3059-3078, 1988.

[39] M. French, M. P. Desjarlais, and R. Redmer, "Ab initio calculation of thermodynamic potentials and entropies for superionic water," Physical Review E, vol. 93, Article ID 022140, 2016.

[40] M. D. Knudson, M. P. Desjarlais, R. W. Lemke et al., "Probing the interiors of the ice giants: shock compression of water to $700 \mathrm{GPa}$ and $3.8\left(\mathrm{~g} / \mathrm{cm}^{3}\right)$," Physical Review Letters, vol. 108, Article ID 091102, 2012.

[41] G. A. Lyzenga, T. J. Ahrens, W. J. Nellis, and A. C. Mitchell, "The temperature of shock-compressed water," The Journal of Chemical Physics, vol. 76, no. 12, pp. 6282-6286, 1982.

[42] M. Guarguaglini, J.-A. Hernandez, T. Okuchi et al., "Laserdriven shock compression of "synthetic planetary mixtures" of water, ethanol, and ammonia," Scientific Reports, vol. 9, no. 1, Article ID 10155, 2019.

[43] D. Batani, K. Jakubowska, A. Benuzzi-Mounaix et al., "Refraction index of shock compressed water in the megabar pressure range," EPL (Europhysics Letters), vol. 112, no. 3, Article ID 36001, 2015.

[44] S. Bossi, T. A. Hall, M. Mahdieh et al., "Determination of the color temperature in laser-produced shocks," Laser and Particle Beams, vol. 15, no. 4, pp. 485-493, 1997.

[45] T. Sano, N. Ozaki, T. Sakaiya et al., "Laser-shock compression and hugoniot measurements of liquid hydrogen to $55 \mathrm{GPa}$," Physical Review B: Condensed Matter, vol. 83, Article ID 054117, 2011.

[46] S. Faik, A. Tauschwitz, and I. Iosilevskiy, "The equation of state package FEOS for high energy density matter," Computer Physics Communications, vol. 227, pp. 117-125, 2018. 